\title{
First Description of the Causal Agent of Taproot Decline of Soybean, an Emerging Disease in the Southern United States
}

\begin{abstract}
Tom Allen, Delta Research and Extension Center, Stoneville, MS 38776; Burt Bluhm, University of Arkansas, Fayetteville 72701; Kassie Conner, Auburn University, Auburn, AL 36849; Vinson Doyle, LSU AgCenter, Department of Plant Pathology and Crop Physiology, Baton Rouge, LA 70803; Trey Price, LSU AgCenter, Macon Ridge Research Station, Winnsboro, LA 71295; Edward Sikora, Auburn University, Auburn, AL 36849; Raghuwinder Singh, LSU AgCenter, Department of Plant Pathology and Crop Physiology, Baton Rouge, LA 70803;

Terry Spurlock, University of Arkansas System Cooperative Extension Service, Southeast Research and Extension Center, Monticello 71656; Maria Tomaso-Peterson, Mississippi State University, Starkville 39762; and Tessie Wilkerson, Delta Research and Extension Center, Stoneville, MS 38776
\end{abstract}

Accepted for publication 8 March 2017.

\section{Abstract}

Over the past decade, a soybean root issue of unknown etiology has been observed across a widespread geography in the southern United States. Recently, pathologists began referring to the problem as taproot decline of soybean, based on the appearance of root symptoms. Taproot decline has been observed to cause foliar symptoms in vegetative and reproductive soybean plants ranging in maturity from V6 to R6. Symptom expression can appear similar to other notable root-associated diseases except that taproot decline exhibits a progression of symptom expression from subtle to severe interveinal chlorosis during the season. However, one distinct symptom associated with taproot decline is observed as darkened, black stroma on the taproot and, in some cases, the lateral roots of affected plants. Pathogenicity was confirmed by isolating the suspected fungus from naturallyinfected soybean roots in multiple states and completing Koch's postulates. The causal agent was identified, based on morphological characters and multilocus phylogenetic inference, as a member of the Xylaria arbuscula aggregate. At present, research projects are underway to address the role of the newly described disease and extent of the pathogen in the southern soybean production region in addition to developing integrated strategies for managing the disease.
Soybean is one of the most important oilseed crops in 70 countries (Hartman et al. 2015). Over the past five growing seasons in Alabama, Arkansas, Louisiana, Mississippi, and Missouri, approximately 5.7 million hectares of soybean were grown annually with crop values ranging from $\$ 5.3$ to $\$ 6.3$ billion per year (Quick Stats, USDA-NASS). In the southern United States, many soilborne pathogens affect the soybean root system throughout the growing season. These diseases include: charcoal rot (caused by Macrophomina phaseolina (Tassi) Goidanich), Phytophthora root rot (caused by Phytophthora sojae Kauf. \& Gerd.), red crown rot (caused by Cylindrocladium crotalariae (Loos) D.K. Bell \& Sobers), root dysfunction caused by southern root-knot nematode (Meloidogyne incognita (Kofoid \& White)), southern blight (caused by Sclerotium rolfsii Sacc.), sudden death syndrome (SDS; caused by Fusarium virguliforme O'Donnell \& T. Aoki), and southern stem canker (caused by Diaporthe aspalathi), which taken collectively as a rootdisease complex caused significant losses during the 2015 growing season (Allen et al. 2016). Many of these soilborne diseases result in foliar symptoms consisting of interveinal chlorosis and subsequent necrosis that are difficult to distinguish from one another or from nutritional imbalances based on foliar symptoms alone. However, in general, observations of nutrient imbalances encompass larger areas in fields where the diseases outlined above, in most cases, affect smaller groups of plants.

Corresponding author: Trey Price; E-mail: pprice@agcenter.lsu.edu

(c) 2017 The American Phytopathological Society
Over the past 10 to 15 years, a malady most often referred to as the "mystery disease" or improperly diagnosed as black root rot (caused by Thielaviopsis basicola) has been encountered in soybean fields in the mid-southern United States and more recently in the southeastern United States. Popular press publications as well as posts on university row-crop blogs (e.g., the Mississippi Crop Situation blog) have broadly referred to the disease. Foliar symptoms associated with the disease have been observed to be nearly identical to the aforementioned soilborne diseases (Price et al. 2015; Tomaso-Peterson et al. 2016). More recently, the malady has been referred to as taproot decline by mid-southern plant pathologists because the symptoms appear to progress on roots and shoots throughout the season from vegetative through reproductive stages of soybean (Price et al. 2015; Tomaso-Peterson et al. 2016).

Since the disease was initially observed by southern soybean pathologists sometime around 2007, several attempts were made to isolate and identify the causal organism. Therefore, the objective of the current study was to isolate, identify, and confirm pathogenicity of the suspected causal agent of taproot decline.

\section{Symptoms of Taproot Decline}

Taproot decline has been confirmed on soil textures varying from sand to clay, and may be observed from early vegetative (V6) to full seed (R6) soybean growth stages throughout the growing season in the southern United States. Unlike some of the aforementioned soybean diseases, the plants expressing symptoms of taproot decline are most often sporadically distributed throughout soybean fields, but can be linearly clustered within the planted furrow and 
are more easily observed during seed filling growth stages as interveinal chlorosis and necrosis occurring on leaves throughout the canopy (Fig. 1). However, foliar symptoms may manifest during vegetative stages with the appearance of subtle interveinal chlorosis

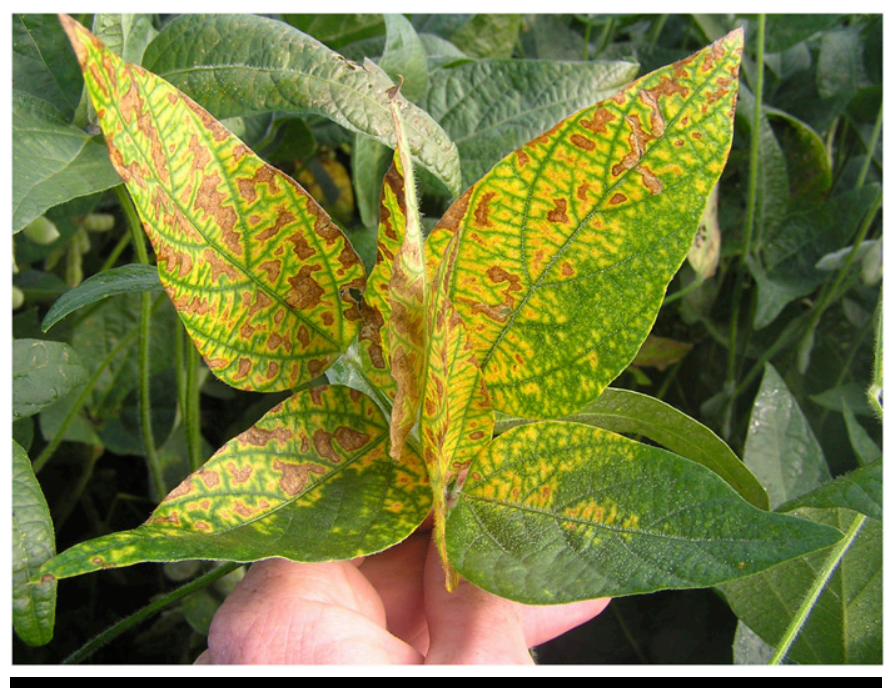

FIGURE 1

Interveinal chlorosis and necrosis caused by taproot decline as observed on R5 to R6 soybean. in the lower plant canopy (Fig. 2A). Most importantly, the severity of interveinal chlorosis increases throughout the season from mild/ subtle symptoms (vegetative) to more severe interveinal chlorosis (late reproductive). Closer examination of soybean plants in the row adjacent to those exhibiting foliar symptoms may reveal smaller plants that have died during seedling, vegetative, or reproductive stages (Fig. 3). During advanced reproductive growth stages ( $>$ R5.5), affected plants, if removed from the ground by grasping the main stem and pulling, break at the soil line, leaving the bulk of the affected roots below the soil surface. When plants are excavated, tap and lateral root surfaces exhibit a blackened appearance (Fig. 4). Subsequent microscopic F4 examination of blackened roots reveals stroma (Fig. 5H). Often, F5 stromata are observed on soybean debris from previous seasons within the furrow or in areas where the plant residue collects as the result of irrigation or spring flooding (Figs. $5 \mathrm{~J}$ and $\mathrm{K})$. Plants, split longitudinally near the crown, often exhibit white, cottony mycelial growth within the pith as well as a mild vascular staining similar to SDS, red crown rot, and southern blight (Fig. 6).

\section{Isolation and Culture of the Pathogen}

A 10-acre field of soybean planted to the cultivar 'Asgrow 4632' (Monsanto Co., St. Louis, MO), was observed exhibiting symptoms of taproot decline at the Macon Ridge Research Station near Winnsboro, LA, during 2015. The previous crop was soybean, and the field was used for commercial soybean production and small

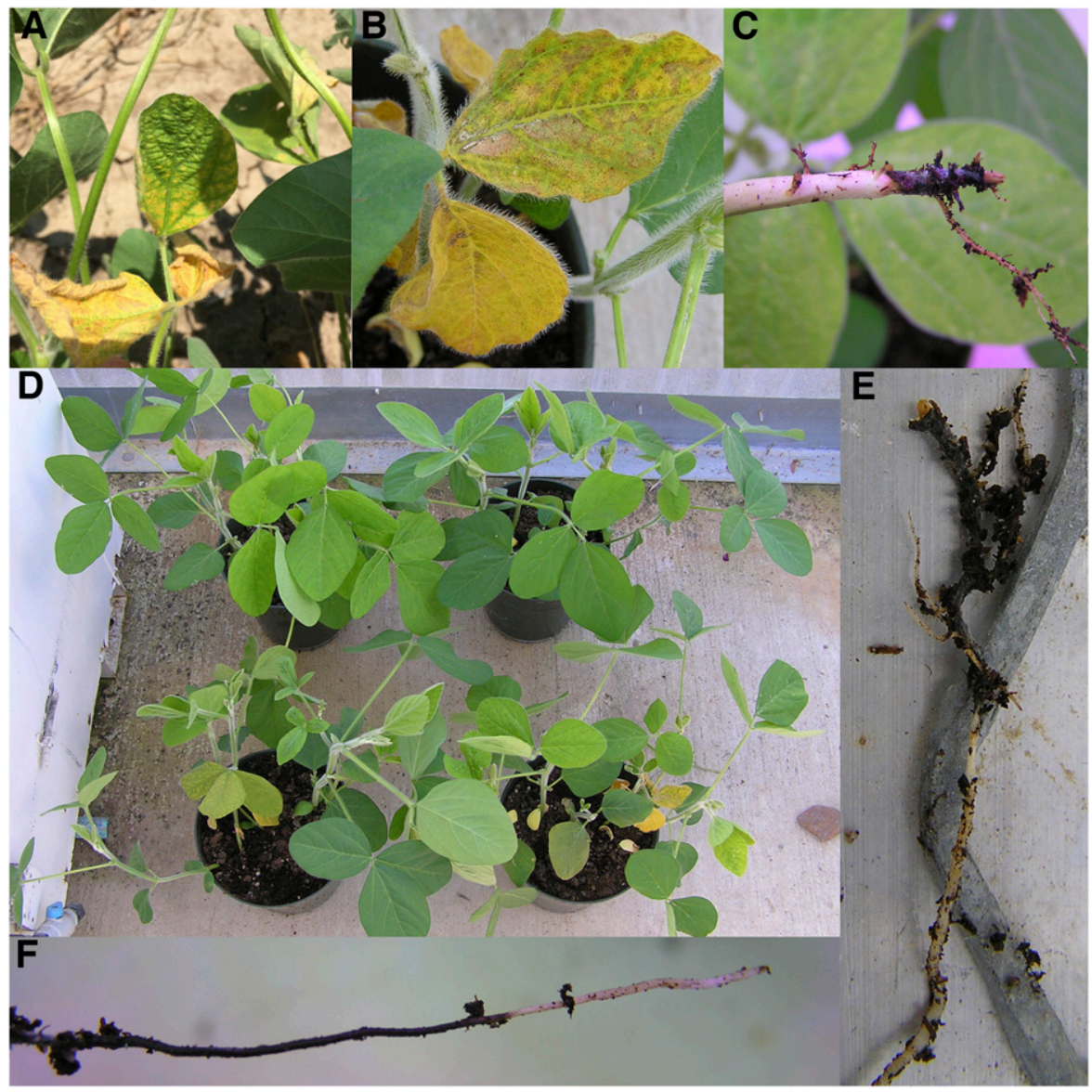

FIGURE 2

Interveinal chlorosis and necrosis caused by taproot decline on $\mathrm{V} 6$ soybean in the field (A) and in the greenhouse (B). Infected tap $\mathbf{C}$ and $\mathbf{E}$ and lateral (F) roots in the greenhouse. Noninoculated (top) versus inoculated (bottom) soybean in the greenhouse (D). 


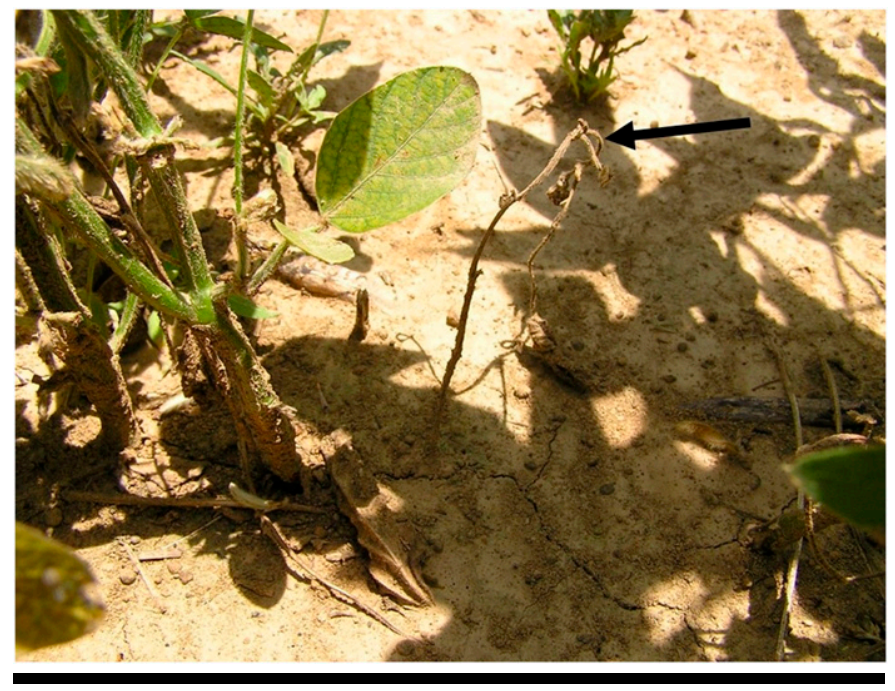

FIGURE 3

Vegetative stage soybean death caused by taproot decline. Arrow indicates the plant that died earlier in the season adjacent to one displaying mild symptoms of interveinal chlorosis (left).

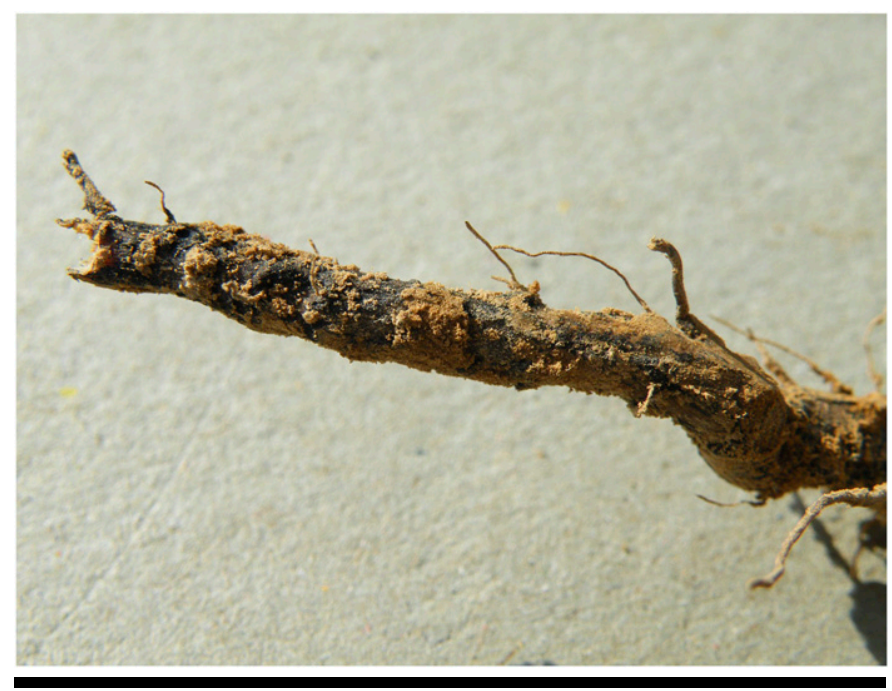

FIGURE 4

Soybean taproot displaying symptoms of taproot decline.

plot research in previous years. Taproot decline incidence in the field was less than $10 \%$. Five plants were excavated from the field and brought to the laboratory. Foliar portions of the plants, cut $7.5 \mathrm{~cm}$ above the crown, were discarded. To remove soil and debris, root crowns, taproots, and lateral roots were rinsed in tap water for $15 \mathrm{~min}$, surface sterilized in a 1:10 sodium hypochlorite solution for $1 \mathrm{~min}$, and again rinsed in tap water for $15 \mathrm{~s}$. A final surface disinfestation of lateral roots ( 2 -cm sections) was accomplished by immersing in 1:10 sodium hypochlorite for 45 to $75 \mathrm{~s}$, rinsing in sterile, distilled water for $1 \mathrm{~min}$, and placing on potato dextrose agar containing chloramphenicol (75 ppm) and streptomycin sulfate (125 ppm) (PDA-CS). Crowns and taproots were split longitudinally under the laminar flow hood with a sterilized scalpel, and white mycelia within the pith were directly transferred to PDA-CS with a sterilized inoculation needle. Mycelial transfers and infested lateral root tissues were incubated at room temperature $\left(20\right.$ to $25^{\circ} \mathrm{C}$ with a 12-h light:dark cycle) for 7 days. Colonies that originated from diseased tissue were selected for subculturing via hyphal tip transfer for subsequent analysis. Recovery of the suspected pathogen occurred $80 \%$ of the time, and fungi not fitting the description below were discarded. Similar isolations of the pathogen exhibiting morphological identity in culture were conducted for suspect roots from all of the states reporting taproot decline as outlined above.

After 14 days of incubation on PDA-CS, colonies that were initially white developed a grayish-black pigment on the surface with black stroma on the reverse (Fig. 5G). Colonies were sterile, circular in form with a starburst growth pattern, and flat with entire margins similar to that observed by Callan and Rogers (1993). Growth rate averaged $4.5 \mathrm{~mm} /$ day. Thin-section bright-field microscopy determined stromata to be sterile. Isolates $(n=9)$ were grown on $2 \%$ oatmeal agar (OA) (Difco; Becton, Dickinson, \& Co., Franklin Lakes, NJ) at $20^{\circ} \mathrm{C}$ (12-h light:dark cycle) to compare with other North American species that have been characterized on the same medium (Callan and Rogers 1993). Growth rates were determined by infesting the center of a $100-\mathrm{mm}$ petri dish containing $25 \mathrm{ml}$ of $2 \%$ OA with a plug taken from the margin of an actively growing culture. Each isolate was plated to three replicates, and three radial measurements were taken from the edge of the plug to the margin of the colony for each plate ( 9 measurements per isolate) every 72 to $96 \mathrm{~h}$ for 21 consecutive days. The majority of isolates covered the plate in less than 3 weeks, and the average growth rate was 1.9 (1.4 to 2.1$) \mathrm{mm} /$ day. As on PDA, colonies were initially white and became gray, gray-brown, or black at maturity (Fig. 5A-C). The colony surface was flocculose to appressed with scalloped to entire margins. The colony reverse was purplish-red pigmented in some isolates (Fig. 5F), but the majority did not exhibit any pigmentation (Fig. 5D). Stromata, similar to that produced by Xylaria spp. (Gonzalez and Rodgers 1989), were frequently produced in culture after several weeks, although not abundantly (Fig. 5I). Most stromata were sterile, but conidia were produced on the colony surface and on the stroma of a few isolates. Conidia were an average of $5.6 \times 2.0 \mu \mathrm{m}$, hyaline, and ellipsoid with a truncate, basal secession scar (Fig. 5L). These characteristics were consistent with the cultural and anamorphic features of Xylaria arbuscula described in the synoptic key provided by Callan and Rogers (1993).

\section{Inoculum Production and Symptom Reproduction}

Stem sections of AsGrow 4632 ( $7.5 \mathrm{~cm}$ long) were collected from residue that originated in 2014 from the field described above and used for inoculum production. Approximately 25 stem sections, each approximately $7.6 \mathrm{~cm}$ long and weighing $10 \mathrm{~g}$ total, were placed in an Erlenmeyer flask along with $100 \mathrm{ml}$ distilled water and autoclaved at $121^{\circ} \mathrm{C}$ for $20 \mathrm{~min}$. After autoclaving, all excess water was aseptically removed from flasks. A 14-day-old colony of the suspected pathogen was aseptically transferred to the flasks (one 1-cm plug per flask), and incubated at $25^{\circ} \mathrm{C}$ with a 12-h light:dark cycle for one month. Stem sections were removed from flasks to the laboratory bench, placed on paper towels, and allowed to dry for 5 days. Soybean seed, AsGrow 4632, were planted (6 seed in 15-cm pots) to Sunshine Professional Growing Mix \#8 (Sun Gro Horticulture, Agawam, MA) supplemented with Osmocote 14-14-14, slow release fertilizer (Everris NA, Inc., Dublin, OH). Prior to planting, grooves 3.5 by $5 \mathrm{~cm}$ deep and wide, respectively, were pressed into the growth medium along the diameter of pots. Seeds were sown evenly across the diameter of the pots. Experimental units were noninoculated, treated with sterilized soybean stems, or 


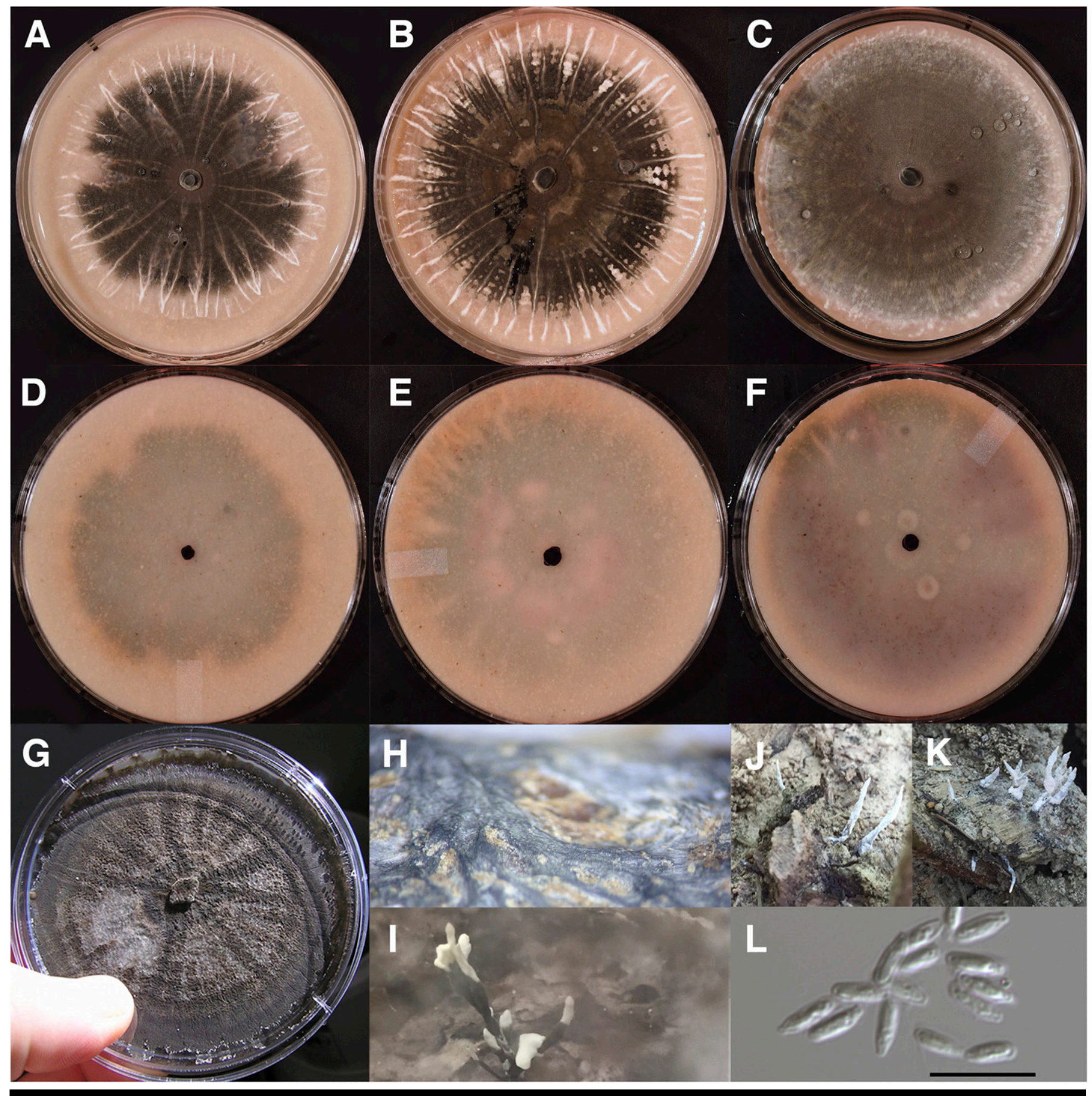

FIGURE 5

Surface $(\mathbf{A}, \mathbf{B}, \mathbf{C})$ and reverse (D, E, F) views of colonies of the taproot decline pathogen on $2 \%$ oatmeal agar. A view of the colony surface (G) of a 14-day-old culture on potato dextrose agar amended with chloramphenicol $(75 \mathrm{ppm})$ and streptomycin sulfate (125 ppm). Stroma (H) and stromata (I) of the taproot decline pathogen in culture. Stromata $(\mathbf{J}, \mathbf{K})$ of the taproot decline pathogen in the field. Conidia of the taproot decline pathogen $(\mathbf{L})(\mathbf{b a r}=10 \mu \mathrm{m})$.

inoculated with colonized soybean stems (one stem section per pot) then covered with growth medium. Pots were flood-irrigated (no direct water contact with the surface of the growth medium) twice daily and grown under supplemental lighting (Welthink LED, Hangzhou, China) with a 12-h light:dark cycle in a greenhouse during the winter months with temperatures ranging from 15.5 to $27^{\circ} \mathrm{C}$. Pots were placed in a randomized complete block design, treatments were replicated three times, and the experiment was repeated twice.
After 3 to 4 weeks, foliar symptoms resembling those as observed on affected vegetative plants in the field (e.g., subtle interveinal chlorosis) developed in plants that were inoculated with colonized stem sections (Fig. 2B). Upon excavation, tap (Figs. 2C and $2 \mathrm{E}$ ) and lateral (Fig. 2F) root sections of inoculated plants were observed to be black in appearance due to the presence of stroma resembling field symptoms. Plants that were not inoculated remained asymptomatic (Fig. 2D). The fungus was reisolated from 
inoculated plants using procedures as previously described. Axenic cultures of the reisolated fungus were identical to those described for the original field isolate. The pathogen was not isolated from noninoculated control plants.

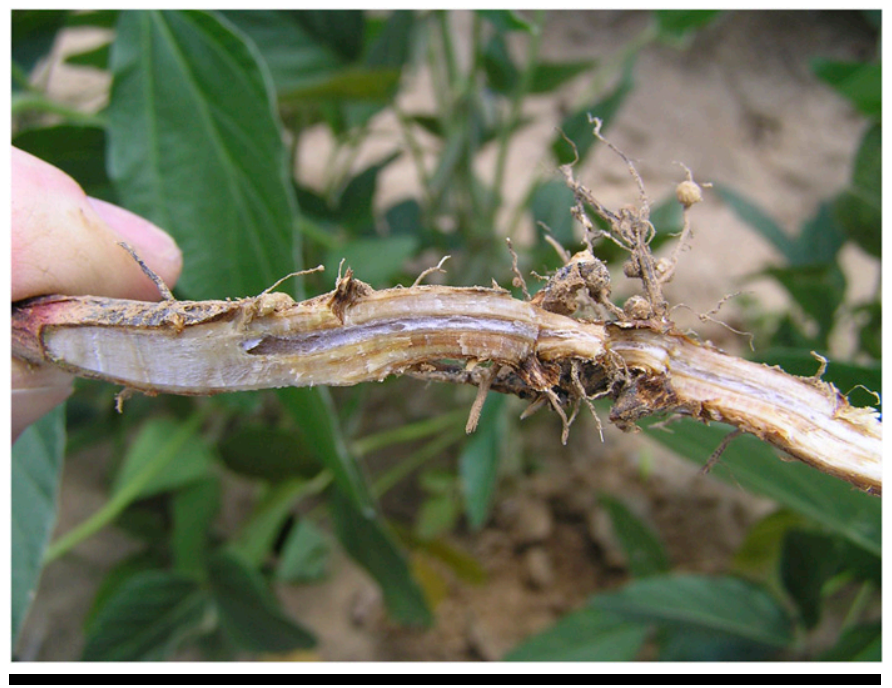

FIGURE 6

White mycelial growth within the pith and vascular staining associated with taproot decline of soybean.

\section{Phylogenetic Characterization}

Initial investigations in 2014 into the identity of the fungus, isolate MSU_SB201401, used the sequence marker of the internal transcribed spacer ITS1-5.8S-ITS2 (ITS) of the rRNA gene amplified with ITS5 and ITS4 (GenBank Accession No. KY433853) (White et al. 1990). The resultant ITS BLASTN revealed $\leq 98 \%$ similarity to Xylaria spp. In order to place the field isolate in a phylogenetic context and make a more precise species assignment, we generated a draft genome sequence of the original field isolate, excised the internal transcribed spacer (ITS; Accession No KY462780), partial alpha-actin (KY646105), partial sequence of the second largest unit of RNA polymerase II (RPB2; KY646106), and partial beta-tubulin (TUB; KY679571), and aligned with homologous reference sequences of Xylaria from previously published studies (Hashemi et al. 2015; Hsieh et al. 2010; Persoh et al. 2009; U'Ren et al. 2016). Each locus was aligned using an iterative refinement strategy (G-INS-i) in MAFFT v. 7 (Katoh and Standley 2013), and poorly aligned regions were removed with Gblocks v 0.91 (Castresana 2000; Talavera and Castresana 2007) using parameters resulting in a less stringent removal of alignment columns. The best-fit model of nucleotide evolution was selected for each locus in jModelTest 2 according to Aikake's information criterion, and the maximum likelihood (ML) phylogeny was estimated in Garli 2.01 (Zwickl 2006) from each locus independently as well as the concatenated, partitioned alignment. Node support was estimated from 1,008 bootstrap replicate datasets and support values mapped

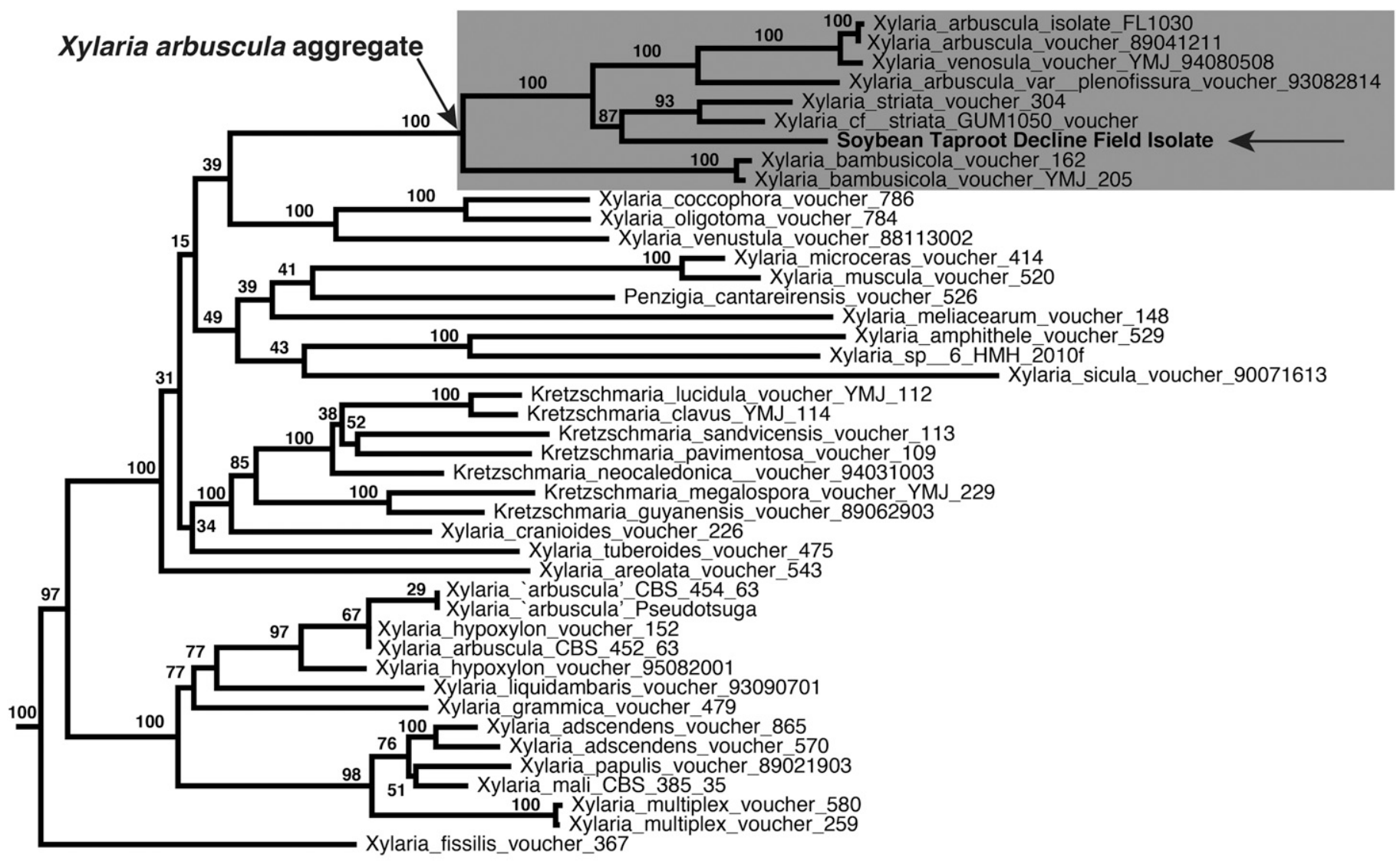

0.06

FIGURE 7

Phylogeny of the taproot decline pathogen. 
to each ML tree. Each individual gene tree (not presented) and the ML tree inferred from the concatenated alignment (Fig. 7) indicate that the causal agent of taproot decline of soybean is a member of the Xylaria arbuscula species aggregate and is strongly supported in the concatenated phylogeny as sister to the sequences representing Xylaria striata Pat. The multiple sequence alignment and tree file are archived at TreeBase (treebase.org, ID 20443). Additional sampling is needed to determine whether the causal agent of taproot decline in soybean represents a novel species or is synonymous with another species within the Xylaria arbuscula species aggregate.

\section{Conclusion and Management Implications}

To our knowledge, this is the first report identifying a causal agent of taproot decline of soybean occurring in the southern United States. In fact, short of a brief report of Xylaria sp. isolated from soybean seed originating in Ethiopia in which disease was not confirmed (Mengistu and Sinclair 1979), this marks the first report of a species of Xylaria causing disease in soybean. Xylaria is principally known to occur in soil and as saprotrophs of wood and other plant parts, but they are also common as endophytes (Petrini and Petrini 1985; U'ren et al. 2016). Few species of Xylaria are reported as pathogens, although Xylaria mali is reported to cause a damaging root rot of apples in the southeastern United States and the Midwest (Rogers 1979; Rogers 1984). Another genus, Kretschmaria, nested within Xylaria in recent phylogenetic studies, contains several species reported to cause butt-rot and root diseases of a wide range of woody angiosperms in the United States and elsewhere (Hsieh et al. 2010; Rogers and Ju 1998; U'ren et al. 2016). Interestingly, all collections of Xylaria striata examined by San Martín et al. (1999), the sister species to the taproot decline isolate of soybean in our phylogeny, observed the stipe of the teleomorph deeply buried in the soil, which suggested it could originate from buried plant material. Rogers (1979) and Rogers and $\mathrm{Ju}$ (1998) suggest that Xylariaceous pathogens are most likely facultative parasites due to their ability to survive on dead plant material, and this may be the mechanism by which taproot decline overwinters in soybean fields.

Taproot decline appears to be mostly distributed throughout the lower Mississippi River Valley; however, the disease has been observed from as far east as the Huntsville, AL, area and as far north as the southeast corner of Missouri. More research involving all aspects of taproot decline of soybean is needed to better understand the pathogen/host relationship and to develop effective management strategies for stakeholders. Currently, anecdotal evidence indicates that taproot decline may cause significant losses (up to 25\%) in areas with reduced tillage and soybean monoculture. A preliminary study conducted in one field in 2014, relying on hand-harvested plots in a commercial field of Asgrow 4632 to compare differences between asymptomatic plants and those exhibiting taproot decline symptoms, indicated that losses of 14 to $26 \%$ were possible in severe situations (unpublished data). Surveys to consider the yield losses associated with taproot decline on a larger scale are needed to assess the potential impacts of this newly emerging root disease on the soybean industry.

\section{Acknowledgments}

The authors would like to thank crop consultants in Mississippi, specifically Brian "Bozo" Ward and Trent LaMastus for bringing this issue to our attention. Without their keen eyes we would not have known about this particular plant disease. Mr. Cliff Coker, Dr. Willam F. Moore, and Dr. Boyd Padgett are appreciated for their preliminary work and photography. In addition, Mr. David and Mr. Tyler Hydrick in northeast Arkansas, and Ms. Donna Lee,
Mr. Bruce Garner, Mr. Ross Bell, Mr. Jimmy House, Mr. Hank Jones, Mr. Todd Knight, Mr. Ashley Peters, and Mr. Chris Ward, in Louisiana, have helped locate affected fields aiding in defining the geographic distribution of the disease. Funding for a portion of this project was graciously provided by the Louisiana Soybean and Grain Research and Promotion Board and Mississippi Soybean Promotion Board. This work was partially supported by the USDA National Institute of Food and Agriculture, Hatch Project LAB94338.

\section{Literature Cited}

Allen, T. W., Damicone, J. P., Dufault, N. S., Faske, T. R., Hershman, D. E., Hollier, C. A., Isakeit, T., Kemerait, R. C., Kleczewski, N. M., Koennig, S. R., Mehl, H. L., Mueller, J. D., Overstreet, C., Price, P., Sikora, E. J., and Young, H. 2016. Southern soybean disease loss estimates for 2015. Page 1-5 in: Proc. of the Southern Soybean Disease Workers $43^{\text {rd }}$ Annual Meeting, Pensacola, FL. March 9-10, 2016.

Callan, B. A., and Rogers, J. D. 1993. A synoptic key to Xylaria species from continental United States and Canada based on cultural and anamorphic features. Mycotaxon 46:141-154.

Castresana, J. 2000. Selection of conserved blocks from multiple alignments for their use in phylogenetic analysis. Mol. Biol. Evol. 17:540-552.

Gonzalez, F. S. M., and Rodgers, J. D. 1989. A preliminary account of Xylaria of Mexico. Mycotaxon 34:283-373.

Hartman, G. L., Rupe, J. C., Sikora, E. J., Domier, L. L., Davis, J. A., and Steffey, K. L. 2015. Compendium of Soybean Diseases and Pests, 5th Ed. American Phytopathological Society, St. Paul, MN.

Hashemi, S. A., Zare, R., Khodaparast, S. A., and Elahinia, S. A. 2015. A new species of Xylaria from Iran. Mycol. Iran. 2:1-10.

Hsieh, H. M., Lin, C. R., Fang, M. J., Rogers, J. D., Fournier, J., Lechat, C., and Ju, Y. M. 2010. Phylogenetic status of Xylaria subgenus Pseudoxylaria among taxa of the subfamily Xylarioideae (Xylariaceae) and phylogeny of the taxa involved in the subfamily. Mol. Phylogenet. Evol. 54:957-969.

Katoh, K., and Standley, D. M. 2013. MAFFT Multiple Sequence Alignment Software Version 7: Improvements in Performance and Usability. Mol. Biol. Evol. 30:772-780.

Mengistu, A., and Sinclair, J. B. 1979. Seedborne microorganisms of Ethiopiangrown soybean and chickpea seeds. Pl. Dis. Rep. 63:616-619.

Persoh, D., Melcher, M., Graf, K., Fournier, J., Stadler, M., and Rambold, G. 2009. Molecular and morphological evidence for the delimitation of Xylaria hypoxylon. Mycologia 101:256-268.

Petrini, L., and Petrini, O. 1985. Xylariaceous fungi as endophytes. Sydowia 38: 216-234.

Price, P., Purvis, M. A., and Pruitt, H. N. 2015. Soybean disease management issues in Louisiana during 2014. Page 24 in: Proc. of the Southern Soybean Disease Workers 42 ${ }^{\text {nd }}$ Annual Meeting, Pensacola, FL. March 11-12, 2015.

Rogers, J. D. 1979. The Xylariaceae: systematic, biological and evolutionary aspects. Mycologia 71:1-42.

Rogers, J. D. 1984. Xylaria acuta, Xylaria cornu-damae, and Xylaria mali in continental United States. Mycologia 76:23-33.

Rogers, J. D., and Ju, Y.-M. 1998. The genus Kretzchmaria. Mycotaxon 68: 345-393.

San Martín, F., Lavín, P., Esqueda-Valle, M., and Pérez-Silva, E. 1999. Additions to known Xylariaceae (Hymenoascomycetes, Xylariales) of Sonora, Mexico. Mycotaxon 70:77-82.

Talavera, G., and Castresana, J. 2007. Improvement of phylogenies after removing divergent and ambiguously aligned blocks from protein sequence alignments. Syst. Biol. 56:564-577.

Tomaso-Peterson, M., Allen, T. W., Price, P., Singh, R., and Spurlock, T. N. 2016. Characterization of taproot decline in southern soybean. Page 19 in: Proc. of the Southern Soybean Disease Workers $43^{\text {rd }}$ Annual Meeting, Pensacola, FL. March 9-10, 2016.

U'Ren, J. M., Miadlikowska, J., Zimmermana, N. B., Lutzoni, F., Stajich, J. E., and Arnold, A. E. 2016. Contributions of North American endophytes to the phylogeny, ecology, and taxonomy of Xylariaceae (Sordariomycetes, Ascomycota). Mol. Phylogenet. Evol. 98:210-232.

White, T. J., Bruns, T., Lee, S., and Taylor, J. W. 1990. Amplification and direct sequencing of fungal ribosomal RNS genes for phylogenetics. Page 315-322 in: PCR Protocols: A Guide to Methods and Applications. M. A. Innis, D. H. Gelfand, J. J. Sninsky, and T. White, eds. Academic Press, San Diego, CA.

Zwickl, D. J. 2006. Genetic Algorithm Approaches for the Phylogenetic Analysis of Large Biological Sequence Datasets Under the Maximum Likelihood Criterion. PhD. dissertation. University of Texas, Austin. 